\title{
PMBH Theory of Representation of Gravity Field Equation and Solutions, Hawking Radiation in Data General Relativity Theory
}

\author{
Sangwha-Yi \\ Department of Math, Taejon University 300-716
}

*Corresponding Author: Sangwha-Yi, Department of Math, Taejon University 300-716

Abstract: In the general relativity theory, we find the representation of the gravity field equation and solutions. We treat the representation of Schwarzschild solution, Reissner-Nodstrom solution, Kerr-Newman solution, Robertson -Walker solution. We found new general relativity theory (we call it Data General Relativity Theory; DGRT). We treat the data of Hawking radiation by Data general relativity theory. This theory has to apply black hole (specially, Primordial Massive Black Hole; PMBH) because black hole $(P M B H)$ is an idealistic structure.

Keywords: General relativity theory, the other solution, Schwarzschild solution, Reissner- Nodstrom solution, Kerr-Newman solution, Robertson-Walker solution, Data General relativity theory, Hawking radiation, Primordial Massive Black Hole

PACS Number: 04, 04.90. +e

\section{INTRODUCTION}

In the general relativity theory, our article's aim is that we find the representation of the gravity field equation and solutions. We found new general relativity theory (we can call it Data General relativity theory). We more obtain data of Hawking radiation by Data general relativity theory.

First, the gravity potential $g_{\mu \nu}$ is

$d s^{2}=g_{\mu \nu} d x^{\mu} d x^{\nu}$

In gravity potential $g_{\mu \nu}$, we introduce tensor $f_{\mu \nu}$ and scalar $K$.

In this time, the condition is

$$
\frac{\partial K}{\partial x^{\lambda}}=0
$$

i) If $g_{00}=-1, f_{\mu \nu}=g_{\mu \nu}, K=1$

ii) If $g_{00} \neq-1, f_{\mu v}=K g_{\mu v}$

Hence,

$$
\begin{aligned}
d s^{R} & =f_{\mu \nu} d x^{\mu} d x^{v}=\bar{g}_{\mu \nu} d \bar{x}^{\mu} d \bar{x}^{v}=\bar{g}_{\mu \nu} \frac{\partial \bar{x}^{\mu}}{\partial \bar{x}^{1 \alpha}} \frac{\partial \bar{x}^{v}}{\partial \bar{x}^{\prime \beta}} d \bar{x}^{1 \alpha} d \bar{x}^{1 \beta} \\
& =\bar{g}_{\alpha \beta}^{\prime} d \bar{x}^{1 \alpha} d \bar{x}^{\prime \beta}=f_{\alpha \beta}^{\prime} d x^{1 \alpha} d x^{\prime \beta} \\
\bar{g}_{\alpha \beta}^{\prime} & =\bar{g}_{\mu \nu} \frac{\partial \bar{x}^{\mu}}{\partial \bar{x}^{\prime \alpha}} \frac{\partial \bar{x}^{v}}{\partial \bar{x}^{\prime \beta}}, g_{\mu \nu}=\bar{g}_{\mu \nu}, g_{\alpha \beta}^{\prime}=\bar{g}_{\alpha \beta}^{\prime} \\
d \bar{x}^{\mu} & =\sqrt{K} d x^{\mu}, d \bar{x}^{v}=\sqrt{K} d x^{v},
\end{aligned}
$$


$d \bar{x}^{1 \alpha}=\sqrt{K} d x^{1 \alpha}, d \bar{x}^{1 \beta}=\sqrt{K} d x^{1 \beta}$

In Christoffel symbol $\Gamma_{\mu \nu}^{\rho}$,

$\bar{\Gamma}_{\mu \nu}^{\rho}=\frac{1}{2} \bar{g}^{\rho \lambda}\left(\frac{\partial \bar{g}_{\mu \lambda}}{\partial \bar{x}^{v}}+\frac{\partial \bar{g}_{\nu \lambda}}{\partial \bar{x}^{\mu}}-\frac{\partial \bar{g}_{\mu v}}{\partial \bar{x}^{\lambda}}\right)=\frac{1}{\sqrt{K}} \Gamma_{\mu \nu}^{\rho}$

Therefore, in the curvature tensor $R^{\rho}{ }_{\mu \nu \lambda}$,

$$
\begin{aligned}
& \bar{R}_{\mu \nu \lambda}{ }_{\mu \nu}=\frac{\partial \bar{\Gamma}_{\mu \nu}^{\rho}}{\partial \bar{x}^{\lambda}}-\frac{\partial \bar{\Gamma}_{\mu \lambda}^{\rho}}{\partial \bar{x}^{\nu}}+\bar{\Gamma}_{\mu \nu}^{\sigma} \bar{\Gamma}_{\sigma \lambda}^{\rho}-\bar{\Gamma}_{\mu \lambda}^{\sigma} \bar{\Gamma}_{\sigma \nu}^{\rho} \\
& =\frac{1}{K}\left(\frac{\partial \Gamma^{\rho}{ }_{\mu \nu}}{\partial x^{\lambda}}-\frac{\partial \Gamma^{\rho}{ }_{\mu \lambda}}{\partial x^{\nu}}+\Gamma_{\mu \nu}^{\sigma} \Gamma_{\sigma \lambda}^{\rho}-\Gamma_{\mu \lambda}^{\sigma} \Gamma_{\sigma \nu}^{\rho}\right)=\frac{1}{K} R^{\rho}{ }_{\mu \nu \lambda}
\end{aligned}
$$

In Ricci tensor $R_{\mu v}$,

$$
\bar{R}_{\mu \nu}=\bar{R}_{\mu \rho v}^{\rho}=\frac{1}{K} R_{\mu \rho \nu}^{\rho}=\frac{1}{K} R_{\mu \nu}
$$

In curvature scalar $R$

$$
\bar{R}=\bar{g}^{\mu v} \bar{R}_{\mu v}=\frac{1}{K} g^{\mu v} R_{\mu v}=\frac{1}{K} R
$$

Hence, in the gravity field equation of Einstein,

$$
\begin{aligned}
& \bar{R}_{\mu \nu}-\frac{1}{2} \bar{g}_{\mu \nu} \bar{R}=\frac{1}{K}\left(R_{\mu \nu}-\frac{1}{2} g_{\mu \nu} R\right) \\
& =-\frac{8 \pi G}{c^{4}} \frac{1}{K} T_{\mu \nu}
\end{aligned}
$$

In Newtonian approximation, Energy-momentum tensor $T_{\mu \nu}$ is

$$
\begin{aligned}
& \bar{\nabla}^{2} \bar{g}_{00}=\frac{1}{K} \nabla^{2} g_{00} \approx-\frac{8 \pi G}{c^{4}} \frac{1}{K} T_{00}=-\frac{8 \pi G}{c^{4}} \bar{T}_{00} \\
& \rho c^{2}=T_{00}, \frac{1}{K} \rho c^{2}=\bar{T}_{00}
\end{aligned}
$$

Hence,

$$
\frac{1}{K} T_{\mu v}=\bar{T}_{\mu v}
$$

Therefore, revised Einstein's gravity field equation is

$$
\bar{R}_{\mu \nu}-\frac{1}{2} \bar{g}_{\mu} \bar{R}=\frac{1}{K}\left(R_{\mu \nu}-\frac{1}{2} g_{\mu} R\right)=-\frac{1}{K} \frac{8 \pi G}{c^{4}} T_{\mu \nu}=-\frac{8 \pi G}{c^{4}} \bar{T}_{\mu \nu}
$$

Therefore, revised gravity field equation of tensor $\bar{g}_{\mu \nu}$ is able to reduce Einstein's gravity field equation. 
Therefore,

$$
\begin{aligned}
& \bar{g}^{\mu \nu}\left[\bar{R}_{\mu \nu}-\frac{1}{2} \bar{g}_{\mu \nu} \bar{R}\right]=\frac{1}{K} g^{\mu \nu}\left[R_{\mu \nu}-\frac{1}{2} g_{\mu \nu} R\right]=-\frac{8 \pi G}{c^{4}} \frac{1}{K} g^{\mu \nu} T_{\mu \nu}=-\frac{8 \pi G}{c^{4}} \frac{1}{K} T_{\lambda}^{\lambda} \\
& =-\frac{8 \pi G}{c^{4}} \bar{g}^{\mu \nu} \bar{T}_{\mu \nu}=-\frac{8 \pi G}{c^{4}} \bar{T}_{\lambda}^{\lambda} \\
& \rightarrow-\bar{R}=-\frac{1}{K} R=-\frac{8 \pi G}{c^{4}} \frac{1}{K} T_{\lambda}^{\lambda}=-\frac{8 \pi G}{c^{4}} \bar{T}_{\lambda}^{\lambda}
\end{aligned}
$$

Hence,

$$
\frac{1}{K} T_{\lambda}^{\lambda}=\bar{T}_{\lambda}^{\lambda}
$$

Ricci tensor is

$\bar{R}_{\mu \nu}=\frac{1}{K} R_{\mu \nu}=-\frac{8 \pi G}{c^{4}} \frac{1}{K}\left(T_{\mu \nu}-\frac{1}{2} g_{\mu \nu} T_{\lambda}^{\lambda}\right)=-\frac{8 \pi G}{c^{4}}\left(\bar{T}_{\mu \nu}-\frac{1}{2} \bar{g}_{\mu \nu} \bar{T}_{\lambda}^{\lambda}\right)$

The proper distance is

$d s^{2}=g_{\mu \nu} d x^{\mu} d x^{\nu}, d s^{R}=f_{\mu \nu} d x^{\mu} d x^{\nu}=\bar{g}_{\mu \nu} d \bar{x}^{\mu} d \bar{x}^{\nu}$

\section{WEAK GRAVITY FIELD APPROXIMATION}

Weak gravity field approximation is

$$
\begin{aligned}
& g_{\mu \nu}=\eta_{\mu \nu}+h_{\mu \nu},\left|h_{\mu \nu}\right|<<1 \\
& R_{\mu \nu}=-\frac{8 \pi G}{c^{4}}\left(T_{\mu \nu}-\frac{1}{2} g_{\mu \nu} T_{\lambda}^{\lambda}\right)
\end{aligned}
$$

According to Eq (15),

$$
\begin{aligned}
& \bar{g}_{\mu \nu}=\bar{\eta}_{\mu \nu}+\bar{h}_{\mu \nu},\left|\bar{h}_{\mu \nu}\right|<<1 \\
& \bar{R}_{\mu \nu}=-\frac{8 \pi G}{c^{4}}\left(\bar{T}_{\mu \nu}-\frac{1}{2} \bar{g}_{\mu \nu} \bar{T}_{\lambda}{ }_{\lambda}\right)
\end{aligned}
$$

The tensor of weak gravity field is

$$
\begin{aligned}
& R_{\mu v} \approx-\frac{8 \pi G}{c^{4}} S_{\mu v}, \bar{R}_{\mu v} \approx-\frac{8 \pi G}{c^{4}} \bar{S}_{\mu v} \\
& S_{\mu v}=T_{\mu v}-\frac{1}{2} \eta_{\mu v} T_{\lambda}^{\lambda}, \\
& \bar{S}_{\mu v}=\bar{T}_{\mu v}-\frac{1}{2} \bar{\eta}_{\mu v} \bar{T}_{\lambda}^{\lambda}
\end{aligned}
$$

The solution is

$$
h_{\mu \nu}(t, \vec{x})=\frac{4 G}{c^{2}} \int d^{4} x^{\prime} \frac{S_{\mu \nu}\left(t-\left|\vec{x}-\vec{x}^{\prime}\right|, \vec{x}^{\prime}\right)}{\left|\vec{x}-\vec{x}^{\prime}\right|},
$$


$\int d^{3} x T_{00}=M$, otherwise $T_{\mu \nu}=0$

$\bar{h}_{\mu \nu}(\bar{t}, \overrightarrow{\bar{x}})=\frac{4 G}{c^{2}} \int d^{4} \bar{x}^{\prime} \frac{\bar{S}_{\mu \nu}\left(\bar{t}-\left|\overrightarrow{\bar{x}}-\overrightarrow{\bar{x}}^{\prime}\right|, \overrightarrow{\bar{x}}^{\prime}\right)}{\left|\overrightarrow{\bar{x}}-\overrightarrow{\bar{x}}^{\prime}\right|}$,

$\int d^{3} \bar{x} \bar{T}_{00}=\int K \sqrt{K} d^{3} \times \frac{1}{K} T_{00}=\sqrt{K} M=\bar{M}, T_{00}=K \bar{T}_{00}$

otherwise $\frac{1}{K} T_{\mu \nu}=\bar{T}_{\mu \nu}=0$

As

$$
\begin{aligned}
& \bar{h}_{00}(\overrightarrow{\bar{x}}) \approx \frac{4 G}{\bar{r} c^{2}} \int d^{3} \bar{x}^{1}\left[\overline{\bar{T}}_{00}-\frac{1}{2} \bar{T}_{00}\right]=\frac{2 \sqrt{K} G M}{\bar{r} c^{2}}, \\
& \bar{h}_{i j}(\overrightarrow{\bar{x}}) \approx \frac{4 G}{\bar{r} c^{2}} \int d^{3} \bar{x}^{\prime}\left[\frac{1}{2} \delta_{i} \overline{\bar{T}}_{00}\right]=\frac{2 \sqrt{K} G M}{\bar{r} c^{2}} \delta_{i \rho}
\end{aligned}
$$

The proper distance is

$$
-d s^{2}=c^{2} d \tau^{2}=-g_{\mu \nu} d x^{\mu} d x^{v} \approx\left(1-\frac{2 G M}{r c^{2}}\right) c^{2} d t^{2}-\left(1+\frac{2 G M}{r c^{2}}\right) \delta_{i} d x^{i} d x^{j}
$$

The proper distance is in this theory

$$
\begin{aligned}
& -d s^{e}=-K d s^{2}=K c^{2} d \tau^{2}=-K g_{\mu \nu} d x^{\mu} d x^{\nu} \\
& \approx K\left(1-\frac{2 G M}{r c^{2}}\right) c^{2} d t^{2}-K\left(1+\frac{2 G M}{r c^{2}}\right) \delta_{i} d x^{i} d x^{j} \\
& =\left(1-\frac{2 \sqrt{K} G M}{\bar{r} c^{2}}\right) c^{2} d \bar{t}^{2}-\left(1+\frac{2 \sqrt{K} G M}{\bar{r} c^{2}}\right) \delta_{i} d \bar{x}^{i} d \bar{x}^{j} \\
& =\left(1-\frac{2 G \bar{M}}{\bar{r} c^{2}}\right) c^{2} d \bar{t}^{2}-\left(1+\frac{2 G \bar{M}}{\bar{r} c^{2}}\right) \delta_{i} d \bar{x}^{j} d \bar{x}^{j} \\
& \approx \bar{g}_{\mu \nu} d \bar{x}^{\mu} d \bar{x}^{v}
\end{aligned}
$$

$$
\sqrt{K} t=\bar{t}, \sqrt{K} x^{i}=\bar{x}^{i}, \sqrt{K} x^{j}=\bar{x}^{j}, \sqrt{K} r=\bar{r}, \sqrt{K} M=\bar{M}
$$

3. THE OTHER REPRESENTATION IN SCHWARZSCHILD SOLUTION, REISSNER-NODSTROM SOLUTION, KERR-NEWMAN SOLUTION AND ROBERTSON-WALKER SOLUTION

Schwarzschild solution (vacuum solution) is

$$
\begin{aligned}
& R_{\mu \nu}=0 \\
& d s^{2}=-c^{2}\left(1-\frac{2 G M}{r c^{2}}\right) d t^{2}+\frac{d r^{2}}{1-\frac{2 G M}{r c^{2}}}+r^{2} d \theta^{2}+r^{2} \sin ^{2} \theta d \phi^{2}
\end{aligned}
$$

The other representation of Schwarzschild solution is 


$$
\begin{aligned}
& d s^{R}=f_{\mu \nu} d x^{\mu} d x^{\nu}=K g_{\mu \nu} d x^{\mu} d x^{\nu}=K \cdot d s^{2} \\
& =-c^{2} K\left(1-\frac{2 G M}{r c^{2}}\right) d t^{2}+\frac{K d r^{2}}{1-\frac{2 G M}{r c^{2}}}+K r^{2} d \theta^{2}+K r^{2} \sin ^{2} \theta d \phi^{2} \\
& =-c^{2}\left(1-\frac{2 \sqrt{K} G M}{\bar{r} c^{2}}\right) d \bar{t}^{2}+\frac{d \bar{r}^{2}}{1-\frac{2 \sqrt{K} G M}{\bar{r} c^{2}}}+\bar{r}^{2} d \bar{\theta}^{2}+\bar{r}^{2} \sin ^{2} \bar{\theta} d \bar{\phi}^{2} \\
& =-C^{2}\left(1-\frac{2 G \bar{M}}{\bar{r} C^{2}}\right) d \bar{t}^{2}+\frac{d \bar{r}^{2}}{1-\frac{2 G \bar{M}}{\bar{r} C^{2}}}+\bar{r}^{2} d \bar{\theta}^{2}+\bar{r}^{2} \sin ^{2} \bar{\theta} d \bar{\phi}^{2}=\bar{g}_{\mu \nu} d \bar{x}^{\mu} d \bar{x}^{\nu} \\
& \sqrt{K} t=\bar{t}, \sqrt{K} r=\bar{r}, \theta=\bar{\theta}, \phi=\bar{\phi}, \sqrt{K} M=\bar{M}
\end{aligned}
$$

Reissner-Nodstrom solution is

$$
\begin{aligned}
& d s^{2}=g_{\mu \nu} d x^{\mu} d x^{\nu} \\
& =-c^{2}\left(1-\frac{2 G M}{r c^{2}}+\frac{k G Q^{2}}{r^{2} c^{4}}\right) d t^{2}+\frac{d r^{2}}{1-\frac{2 G M}{r c^{2}}+\frac{k G Q^{2}}{r^{2} c^{4}}}+r^{2} d \theta^{2}+r^{2} \sin ^{2} \theta d \phi^{2}
\end{aligned}
$$

The other representation of Reissner-Nodstrom solution is

$$
\begin{aligned}
& d s^{R}=f_{\mu \nu} d x^{\mu} d x^{\nu}=K g_{\mu \nu} d x^{\mu} d x^{\nu}=K \cdot d s^{2} \\
& =-K c^{2}\left(1-\frac{2 G M}{r c^{2}}+\frac{k G Q Q^{2}}{r^{2} c^{4}}\right) d t^{2}+\frac{K d r^{2}}{1-\frac{2 G M}{r c^{2}}+\frac{k G Q^{2}}{r^{2} c^{4}}}+K r^{2} d \theta^{2}+K r^{2} \sin ^{2} \theta d \phi^{2} \\
& =-c^{2}\left(1-\frac{2 \sqrt{K} G M}{\bar{r} c^{2}}+\frac{K K G Q Q^{2}}{\bar{r}^{2} c^{4}}\right) d \bar{t}^{2}+\frac{d \bar{r}^{2}}{1-\frac{2 \sqrt{K} G M}{\bar{r} c^{2}}+\frac{K k G Q Q^{2}}{\bar{r}^{2} c^{4}}}+\bar{r}^{2} d \bar{\theta}^{2}+\bar{r}^{2} \sin ^{2} \bar{\theta} d \bar{\phi}^{2}
\end{aligned}
$$

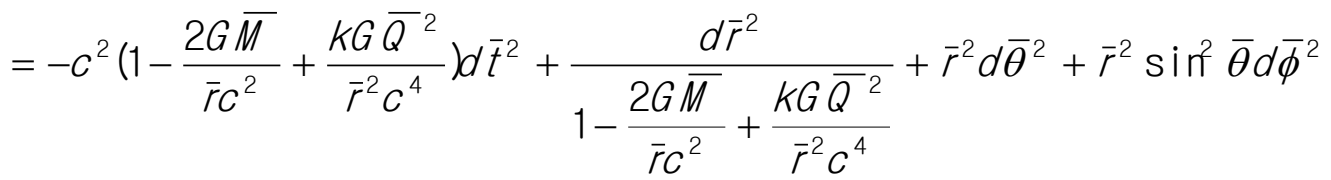

$$
\begin{aligned}
& =\bar{g}_{\mu \nu} d \bar{x}^{\mu} d \bar{x}^{\nu} \\
& \sqrt{K} t=\bar{t}, \sqrt{K} r=\bar{r}, \theta=\bar{\theta}, \phi=\bar{\phi}, \sqrt{K} M=\bar{M}, K Q^{2}=\bar{Q}^{2}
\end{aligned}
$$

Kerr-Newman solution is $d s^{2}=g_{\mu \nu} d x^{\mu} d x^{\nu}$

$$
\begin{aligned}
= & -c^{2}\left(1-\frac{2 c^{2} G M r-k G Q^{2}}{c^{4} \Sigma}\right) d t^{2}+2\left(2 c^{2} M G r-k G Q^{2}\right) \frac{a \sin ^{2} \theta}{c^{4} \Sigma} c d t d \phi \\
& -\frac{c^{4} \Sigma}{r^{2}-c^{2} 2 G M r+a^{2}+k G Q^{2}} d r^{2}-\Sigma d \theta^{2}
\end{aligned}
$$




$$
\begin{aligned}
& -\sin ^{2} \theta\left[r^{2}+a^{2}+\left(2 c^{2} G M r-k G Q 2\right) \frac{a^{2} \sin ^{2} \theta}{c^{4} \Sigma}\right] d \phi^{2} \\
& \Sigma=r^{2}+a^{2} \cos ^{2} \theta
\end{aligned}
$$

The other representation of Kerr-Newman solution is

$$
\begin{aligned}
& d s^{R}=f_{\mu \nu} d x^{\mu} d x^{v}=K g_{\mu \nu} d x^{\mu} d x^{\nu}=K \cdot d s^{2} \\
& =-K c^{2}\left(1-\frac{2 c^{2} G M r-k G Q^{2}}{c^{4} \Sigma}\right) d t^{2}+2 K\left(2 c^{2} M G r-k G Q{ }^{2}\right) \frac{a \sin ^{2} \theta}{c^{4} \Sigma} c d t d \phi \\
& -\frac{K \Sigma c^{4}}{r^{2}-2 c^{2} G M r+a^{2}+k G Q Q^{2}} d r^{2}-K \Sigma d \theta^{2} \\
& -K \sin ^{2} \theta\left[r^{2}+a^{2}+\left(2 c^{2} G M r-k G Q 2\right) \frac{a^{2} \sin ^{2} \theta}{c^{4} \Sigma}\right] d \phi^{2} \\
& =-c^{2}\left(1-\frac{2 c^{2} G \sqrt{K} M \sqrt{K} r-k G K Q^{2}}{K \Sigma c^{4}}\right) d \bar{t}^{2}+2\left(2 c^{2} \sqrt{K} M G \sqrt{K} r-k G K Q^{2}\right) \frac{\sqrt{K} a \sin ^{2} \theta}{K \Sigma c^{4}} c d \sqrt{K} t d \phi \\
& -\frac{K \Sigma c^{4}}{K r^{2}-2 c^{2} G \sqrt{K} M \sqrt{K} r+K a^{2}+k G K Q^{2}} d(\sqrt{K} r)^{2}-K \Sigma d \theta^{2} \\
& -\sin ^{2} \theta\left[K r^{2}+K a^{2}+\left(2 c^{2} G \sqrt{K} M \sqrt{K} r-K G K Q{ }^{2}\right) \frac{K a^{2} \sin ^{2} \theta}{K \Sigma c^{4}}\right] d \phi^{2} \\
& =-c^{2}\left(1-\frac{2 c^{2} G \bar{M} \bar{r}-k G \bar{Q}^{2}}{\bar{\Sigma} c^{4}}\right) d \bar{t}^{2}+2\left(2 c^{2} \bar{M} G \bar{r}-k G \bar{Q}^{2}\right) \frac{\bar{a} \sin ^{2} \bar{\theta}}{\bar{\Sigma} c^{4}} c d \bar{t} d \bar{\phi} \\
& -\frac{\bar{\Sigma} c^{4}}{\bar{r}^{2}-2 c^{2} G \bar{M} \bar{r}+\bar{a}^{2}+k G \bar{Q}^{2}} d \bar{r}^{2}-\bar{\Sigma} d \bar{\theta}^{2} \\
& -\sin ^{2} \bar{\theta}\left[\bar{r}^{2}+\bar{a}^{2}+\left(2 c^{2} G \bar{M} \bar{r}-k G \bar{Q}^{2}\right) \frac{\bar{a}^{2} \sin ^{2} \bar{\theta}}{c^{4} \bar{\Sigma}}\right] d \bar{\phi}^{2} \\
& =\bar{g}_{\mu \nu} d \bar{x}^{\mu} d \bar{x}^{v} \\
& \bar{\Sigma}=K \Sigma=K r^{2}+K a^{2} \cos ^{2} \theta=\bar{r}^{2}+\bar{a}^{2} \cos ^{2} \bar{\theta} \\
& \sqrt{K} t=\bar{t}, \sqrt{K} r=\bar{r}, \theta=\bar{\theta}, \phi=\bar{\phi}, \sqrt{K} M=\bar{M}, K Q^{2}=\bar{Q}^{2}, \sqrt{K} a=\bar{a} \text {, } \\
& \bar{J}=c \bar{M} \bar{a}=K c M a=K J
\end{aligned}
$$

In this time, we obtain the data of the time $t$, the distance $r$, the mass $M$, the charge $Q$ and the angular momentum $\mathrm{J}$.

Robertson-Walker solution is

$$
\begin{aligned}
& d s^{2}=g_{\mu \nu} d x^{\mu} d x^{\nu} \\
& =-c^{2} d t^{2}+\Omega^{2}(t)\left[\frac{d r^{2}}{1-k r^{2}}+r^{2} d \theta^{2}+r^{2} \sin ^{2} \theta d \phi^{2}\right]
\end{aligned}
$$


$k=(-1,0,1)$

The other representation of Robertson-Walker solution is by $\mathrm{Eq}(1-\mathrm{i})$

$$
\begin{aligned}
& d s^{2}=f_{\mu \nu} d x^{\mu} d x^{\nu}=g_{\mu \nu} d x^{\mu} d x^{\nu} \\
& =-c^{2} d \bar{t}^{2}+\bar{\Omega}^{2}(\bar{t})\left[\frac{d \bar{r}^{2}}{1-k \bar{r}^{2}}+\bar{r}^{2} d \bar{\theta}^{2}+\bar{r}^{2} \sin ^{2} \bar{\theta} d \bar{\phi}^{2}\right] \quad \bar{g}_{\mu \nu} d \bar{x}^{\mu} d \bar{x}^{v} \\
& k=(-1,0,1), \quad t=\bar{t}, \quad \Omega(t)=\bar{\Omega}(\bar{t}), r=\bar{r}, \theta=\bar{\theta}, \phi=\bar{\phi}
\end{aligned}
$$

\section{OBTAINING PROCESS INFORMATION OF HAWKING RADIATION}

Stephen Hawking found black -hole's thermodynamics. By Hawking Radiation, we obtain the new data from formulas of black-hole's thermodynamics. We start the obtaining process informations of Hawking Radiation. In Wikipedia (Hawking Radiation), we know formulas of Hawking Radiation.

The radiation temperature $T$ of Schwarzschild black hole (In this theory, PMBH)

$$
T=\frac{\hbar c^{3}}{8 \pi G M k_{B}}
$$

The radiation temperature $\bar{T}$ is in Data General Relativity theory.

$$
\bar{T}==\frac{\hbar c^{3}}{8 \pi G \bar{M} k_{B}}=\frac{\hbar c^{3}}{8 \pi G \sqrt{K} M k_{B}}=\frac{T}{\sqrt{K}}
$$

The black hole (PMBH)'s entropy

$$
\begin{aligned}
& d S=8 \pi G k_{B} M d M \quad / \hbar c=\frac{8 \pi G M k_{B}}{\hbar c^{3}} c^{2} d M=\frac{d Q}{T}, \\
& d Q=c^{2} d M
\end{aligned}
$$

The black-hole (PMBH)'s entropy $\bar{S}$ is in Data General Relativity theory.

$$
\begin{aligned}
& d \bar{S}=8 \pi \bar{M} d \bar{M} G k_{B} / \hbar c=\frac{8 \pi G k_{B} \bar{M}}{\hbar c^{3}} c^{2} d \bar{M}=\frac{d \bar{Q}}{\bar{T}} \\
& =\frac{8 \pi G k_{B} \sqrt{K} M}{\hbar c^{3}} c^{2} d(\sqrt{K} M) \\
& =K \frac{8 \pi G k_{B} M}{\hbar c^{3}} c^{2} d M \\
& =K \frac{d Q}{T}=K d S, d Q=c^{2} d M \\
& d \bar{Q}=c^{2} d \bar{M}=\sqrt{K} c^{2} d M=\sqrt{K} d Q \\
& \bar{S}=K S, \bar{Q}=\sqrt{K} Q
\end{aligned}
$$

Black-hole (PMBH) radiation's power $P_{e v}$ is 


$$
P_{e v}=A_{S} \varepsilon \sigma T^{4}, A_{s}=4 \pi r_{S}^{2}, r_{S}=\frac{2 G M}{c^{2}}, \varepsilon, \sigma \text { is constant }
$$

Black-hole (PMBH) radiation's power $\bar{P}_{e V}$ is in Data General Relativity theory.

$$
\begin{aligned}
& \bar{A}_{s}=4 \pi \bar{r}_{S}^{2}=K 4 \pi r_{S}^{2}=K A_{s}, \quad \bar{r}_{S}=\frac{2 G \bar{M}}{c^{2}}=\frac{2 G \sqrt{K} M}{c^{2}}=\sqrt{K} r_{S} \\
& \bar{P}_{e V}=\bar{A}_{S} \varepsilon \sigma \bar{T}^{4}=K A_{s} \varepsilon \sigma \frac{T^{4}}{K^{2}}=\frac{P_{e V}}{K}
\end{aligned}
$$

Stefan-Boltzmann constant $\sigma=\frac{\pi^{2} k_{B}{ }^{4}}{60 \hbar^{3} c^{2}}$,

Black-hole $(\mathrm{PMBH})$ is a perfect black body $(\varepsilon=1)$

The evaporation time $t_{e v}$ of a black hole $(\mathrm{PMBH})$ is

$$
t_{e v}=\frac{5120 \pi G^{2} M^{3}}{\hbar c^{4}}
$$

The evaporation time $\bar{t}_{e v}$ of a black hole (PMBH) is in Data General Relativity theory.

$$
\bar{t}_{e v}=\frac{5120 \pi G^{2} \bar{M}^{3}}{\hbar c^{4}}=\frac{5120 \pi G^{2} K \sqrt{K} M^{3}}{\hbar c^{4}}=K \sqrt{K} t_{e v}
$$

The power of evaporation energy of the black-hole $(\mathrm{PMBH})$ is

$$
P_{e v}=-\frac{d E_{e v}}{d t_{e v}}
$$

The power of evaporation energy of the black-hole (PMBH) is in Data General Relativity theory.

$$
\begin{aligned}
& \bar{P}_{e v}=-\frac{d \bar{E}_{e v}}{d \bar{t}_{e v}}=-\frac{d\left(E_{e v} \sqrt{K}\right)}{K \sqrt{K} d t_{e v}}=\frac{P_{e v}}{K}, \\
& \bar{M}_{e v} c^{2}=\bar{E}_{e v}=\sqrt{K} E_{e v}=\sqrt{K} M_{e v} c^{2}
\end{aligned}
$$

\section{PMBH DATA AND DGRT DATA}

In DGRT, we have to apply only black-holes (PMBHs).

According to [27] Paul H. Frampton, Physical Letter B (2017), if the mass of sun is $M_{\Theta}$, data is

Table1.

\begin{tabular}{|l|l|l|l|}
\hline Astrophysical obect & Mass solar masses & Period $\tau$ seconds & Angular momentum $\mathrm{kgkm}^{2} / \mathrm{s}$ \\
\hline PIMBH1 & $20 \mathrm{M} \Theta$ & $0.013 \mathrm{~s}$ & $3.0 \times 10^{37}$ \\
\hline PIMBH2 & $100 \mathrm{M} \Theta$ & $0.063 \mathrm{~s}$ & $7.2 \times 10^{38}$ \\
\hline PIMBH3 & $1000 \mathrm{M} \Theta$ & $0.63 \mathrm{~s}$ & $7.2 \times 10^{40}$ \\
\hline PIMBH4 & $10^{4} \mathrm{M} \Theta$ & $6.3 \mathrm{~s}$ & $7.2 \times 10^{42}$ \\
\hline PIMBH5 & $10^{5} \mathrm{M} \Theta$ & $63 \mathrm{~s}$ & $7.2 \times 10^{44}$ \\
\hline PSMBH6(M87) & $6 \times 10^{9} \mathrm{M} \Theta$ & $3.8 \times 10^{6} \mathrm{~s}$ & $2.6 \times 10^{54}$ \\
\hline
\end{tabular}


According to DGRT, BH (PMBH)'s mass $M$ is to be $\sqrt{K} M$, time $\tau$ is to be $\tau \sqrt{K}$, Angular momentum $J$ is to be $K J$.Hence, PIMBH2's (from PIMBH1) $\sqrt{K}$ is 5 , PIMBH3's (from PIMBH 2) $\sqrt{K}$ is $10, \mathrm{PIMBH} 4$ 's(from PIMBH3) $\sqrt{K}$ is 10 , PIMBH5's (from PIMBH4) $\sqrt{K}$ is 10 , PSMBH6's (from PIMBH5) $\sqrt{K}$ is $6 \times 10^{4}$.

In this time, PIMBH is Primordial Intermediate Massive Black hole, PSMBH is Primordial Super Massive Black Hole. According to [27]"Angular momentum of dark matter black holes", $20 M_{\Theta} \leq M_{P / M B H} \leq 100,000 M_{\Theta}$

$10^{5} M_{\Theta} \leq M_{P S M B H} \leq 10^{17} M_{\Theta}$

Therefore, calculated data is in DGRT,

Table2.

\begin{tabular}{|l|l|l|l|}
\hline Astrophysical obect & Mass solar masses & Period $\tau$ seconds & Angular momentum $\mathrm{kgkm}^{2} / \mathrm{s}$ \\
\hline PIMBH1 & $20 \mathrm{M} \Theta$ & $0.013 \mathrm{~s}$ & $3.0 \times 10^{37}$ \\
\hline PIMBH2 & $100 \mathrm{M} \Theta$ & $0.065 \mathrm{~s}$ & $7.5 \times 10^{38}$ \\
\hline PIMBH3 & $1000 \mathrm{M} \Theta$ & $0.65 \mathrm{~s}$ & $7.5 \times 10^{40}$ \\
\hline PIMBH4 & $10^{4} \mathrm{M} \Theta$ & $6.5 \mathrm{~s}$ & $7.5 \times 10^{42}$ \\
\hline PIMBH5 & $10^{5} \mathrm{M} \Theta$ & $65 \mathrm{~s}$ & $7.5 \times 10^{44}$ \\
\hline PSMBH6(M87) & $6 \times 10^{9} \mathrm{M} \Theta$ & $3.9 \times 10^{6} \mathrm{~s}$ & $2.7 \times 10^{54}$ \\
\hline
\end{tabular}

If we compare Table 1. and Table 2., we know DGRT has to apply PMBHs.

\section{CONCLUSION}

We find the other representation of solutions in the General relativity theory. We more obtain the information of black-hole thermodynamics in Data General Relativity theory.

If we use variable $\bar{A}$ instead of $A$, Data General Relativity theory is reduced to normal general relativity theory. This theory's remarkable thing is if $\sqrt{K}=2$ and black hole (PMBH)'s mass $M$ is $M \sqrt{K}=2 M$, black hole (PMBH)'s distant of gravitation $r$ is $r \sqrt{K}=2 r$, black hole (PMBH)'s proper time $\tau$ is $\tau \sqrt{K}=2 \tau$.If rotating black hole (PMBH)'s mass $M$ is to be $M \sqrt{K}=2 M$, we predict the angular momentum $J$ of the black-hole $(\mathrm{PMBH})$ is to be $K J=4 \mathrm{~J}$.

In this time, we have to apply only black-holes (PMBHs) because black hole (PMBH) is an idealistic structure. BH is Black hole.

\section{REFERENCES}

[1] S. Weinberg, Gravitation and Cosmology (John wiley \& Sons,Inc,1972)

[2] R. Eotvos, D. Pekar and E. Fetkete, Ann.Phys.(Germany) 68,11(1922)

[3] C. Misner, K, Thorne and J. Wheeler, Gravitation (W.H.Freedman \& Co.,1973)

[4] S. Hawking and G. Ellis, The Large Scale Structure of Space-Time(Cam-bridge University Press,1973)

[5] R. Adler,M.Bazin and M.Schiffer,Introduction to General Relativity(McGraw-Hill,Inc.,1965)

[6] Hawking Radiation-Wikipedia

[7] P. Roll, R. Krotkov and R. Dicke, Ann. Phys.(U.S.A) 26,442(1964)

[8] V. Braginsky and V. Panov, Zh. Eksp. \& Teor. Fiz.61, 873(1971)(English translation, Sov. Phys.-JETP 34,464(1971)

[9] L.Einsenhart, Riemannian Geometry(Princeton University Press, 1926)

[10] J. Schouten, Ricci-Calculus(Springer-Verlag, Berlin, 1954)

[11] A. Einstein,'The Foundation of General Relativity", Ann. Phys. (Germany)49(1916) 
[12] G.Birkoff, Relativity and Modern Physics (Harvard University Press,1923),p253

[13] A.Raychaudhuri, Theorical Cosmology(Oxpord University Press, 1979)

[14] E. Kasner, Am. J. Math. 43, 217(1921)

[15] D.N.Page."Hawking Radiation and Black hole Thermodynamics.".arXiv:hep-th/040924

[16] M.Rabinowitz."Gravitaational Tunneling Radiation". Physics Essays. 12(2):346-357.arxiv:astro-ph/02 12249(2000)

[17] S,Giddings and S.Thomas "High energy colliders as black hole factories: The end of short distant physics". Physical Review D. 65(5)(2002)

[18] S.Dimoploulus and G.Landsberg "Black holes at the Large Hardron Collider". Physical Review Letter. 87(16):161602.arxiv:hep-th/0106295(2001)

[19] F.Belgiorno ,S.Cacciatori, M.Clerici, V.Gorini, G. Ortenzi, L.Rizzi ,E.Rubbino, V.Sala D. Faccio.” Hawking Radiation from ultrashort laser pulse filaments".Phys. Rev. Lett. 105(20):203901(2010): arxiv:1009. 4634

[20] K. Kumar, B. Kiranagi, C. Begewadi, "Hawking Radiation- An Augmentation Attrition Model”. Adv. Nat. Sci.5.(2):14-33(2012)

[21] A. Helfer "Do black-holes radiate?".Reports on Progress in Physics. 66(6):943-1008(2003):arxiv:grqc/0304042.(2003)

[22] R. Brout, S. Massar, R. Parentani, P. Spindel "Hawking radiation without tras-Plankian frequencies". Phiscal Review D.52(8):4559-4568(1995):arxiv:hep-th/9506121

[23] D. Page, "Particle emission rates from a black-hole: Massless particles from an unchrge, nonrotating hole". Physical Review D.13(2):198-206(1976)

[24] T. Jacobson, "Black hole-evaporation and ultrashorts distances" Physical Review D.44(6):1731-1739 (1991)

[25] J. Kapusta, "The Last Eight Minutes of a Primordial Black-hole" (1999):Arxiv:astro-ph/9911309

[26] A. Ashtekar, J. Baez, A. Corichi, K. Krasnov, "Quantum Geometry and Black Hole Entropy" Phys. Rev. Lett.80(5):904-907(1998):arxiv:gr-qc/9710007

[27] Paul H. Frampton, "Angular momentum of dark matter black holes" Physical Letter B,767 (303306)(2017)

Citation: Sangwha-Yi, "PMBH Theory of Representation of Gravity Field Equation and Solutions, Hawking Radiation in Data General Relativity Theory", International Journal of Advanced Research in Physical Science (IJARPS), vol. 5, no. 9, pp. 36-45, 2018.

Copyright:@ 2018 Authors, This is an open-access article distributed under the terms of the Creative Commons Attribution License, which permits unrestricted use, distribution, and reproduction in any medium, provided the original author and source are credited. 